Pacific

Journal of

Mathematics

A MURASUGI DECOMPOSITION FOR ACHIRAL ALTERNATING LINKS

Cam Van Quach Hongler and Claude Weber

Volume $222 \quad$ No. 2

December 2005 


\title{
A MURASUGI DECOMPOSITION FOR ACHIRAL ALTERNATING LINKS
}

\author{
Cam Van Quach Hongler and Claude Weber
}

\begin{abstract}
We prove that an achiral alternating link can be decomposed in a strong sense as a Murasugi sum of a link and its mirror image. The proof relies on our theory of Murasugi atoms. We introduce a notion of a bond between atoms, called adjacency. This relation is expressed by a graph, the adjacency graph, which is an isotopy invariant. A well-defined link type, called a molecule, is associated to any connected subgraph of the adjacency graph. The Flyping Theorem of Menasco and Thistlethwaite is the main tool used to prove the isotopy invariance of atoms, molecules and the adjacency graph. The action of flypes on the adjacency graph and the invariance of the collection of molecules under flypes are the main ingredients of the proof of the decomposition theorem.
\end{abstract}

\section{Introduction}

A cheap way to produce an achiral knot is to construct the connected sum of a knot $K$ with its mirror image; but it is certainly not true that all achiral knots arise in this way! The main result of this article is that it is true that all alternating achiral knots (in fact, links) come from this procedure, if the notion of connected sum is replaced by the more general notion of Murasugi sum.

As Mikami Hirasawa has kindly pointed out, we must clarify which kind of Murasugi sum we work with. The classical definition of Murasugi sum involves two oriented links $L^{\prime}$ and $L^{\prime \prime}$ in $S^{3}$. An arbitrary choice is made of Seifert surfaces $S^{\prime}$ and $S^{\prime \prime}$ for each link. Then on each surface a $2 n$-gon is chosen: $P^{\prime}$ in $S^{\prime}$ and $P^{\prime \prime}$ in $S^{\prime \prime}$. We select a 2-sphere $S^{2}$ in $S^{3}$ inducing a decomposition of $S^{3}$ in two hemispheres. Then $S^{\prime}$ is moved into one hemisphere and $S^{\prime \prime}$ into the other in such a way that, among other conditions,

$$
P^{\prime}=S^{\prime} \cap S^{2}=S^{\prime \prime} \cap S^{2}=P^{\prime \prime} .
$$

MSC2000: 57M25.

Keywords: alternating links, achiral links, invertible links, Murasugi decomposition, flype conjecture.

We thank the Fonds National Suisse de la Recherche Scientifique for their support. 
See [Gabai 1983] for more details. The abundance of possible choices has several consequences, most of them unwanted. For instance, Thompson [1994] has given an example of two nontrivial knots which can be summed along adequately selected Seifert surfaces (not of minimal genus) to produce the trivial knot. Hirasawa has announced, in a talk given in Geneva in February 2003, that given three oriented knots $K_{0}, K_{1}$ and $K_{2}$ it is always possible to exhibit $K_{0}$ as a Murasugi sum of $K_{1}$ and $K_{2}$. On the other hand, Gabai [1983] has shown that if incompressible (or minimal genus) Seifert surfaces are used, then the resulting surface is again incompressible (or of minimal genus). But even if one decides to restrict the choices by imposing conditions on the Seifert surfaces, too many possibilities remain. It seems hopeless to look for a uniqueness result for Murasugi decompositions in this context. This is why, when we were working on our paper [Quach Hongler and Weber 2004], we decided to depart from Seifert surfaces and to deal with reduced alternating diagrams instead.

A diagrammatic Murasugi sum using these diagrams is defined in the beginning of Section 5, and we denote it by $D^{\prime} * D^{\prime \prime}$. If we apply the Seifert construction, we obtain Seifert surfaces which have (among others) the property of being of minimal genus. We take the liberty of writing $L^{\prime} * L^{\prime \prime}$ for a sum of oriented links obtained from reduced alternating diagrams. In this paper, we shall use only this refined version of Murasugi sums, and phenomena like those discovered by Thompson and Hirasawa are thus impossible.

Notation. If $L$ is an oriented link, $\hat{L}$ denotes the mirror image of $L$ as an oriented link. We write $-L$ for the oriented link obtained from $L$ by reversing the orientations of all its components. An oriented link $L$ is positively achiral if it is isotopic to $\hat{L}$, and negatively achiral if it is isotopic to $-\hat{L}$.

Theorem 5.2. (1) If $L$ is positively achiral, then there exists a link $L^{\prime}$ such that $L=L^{\prime} * \hat{L}^{\prime}$.

(2) If $L$ is negatively achiral, then there exists a link $L^{\prime}$ such that $L=L^{\prime} *-\hat{L}^{\prime}$.

The proof rests on our theory of Murasugi atoms [Quach Hongler and Weber 2004]. In Section 2, we quickly recall basic facts about atoms, and then introduce a notion of bond between atoms, which we call adjacency. Adjacency is a binary relation among atoms of a given link $L$ which can be expressed by the adjacency graph $\Pi(L)$. Its vertices are labeled by the various atoms of $L$ and an edge connects two vertices if the corresponding atoms are adjacent. The graph is constructed from a reduced diagram $D$ representing $L$, and the key point is to prove that the adjacency relation does not depend on the choice of $D$. The Flyping Theorem of Menasco and Thistlethwaite [1993] is crucial here. As a consequence, the graph $\Pi(L)$ is an isotopy invariant. This is proved in Theorem 2.7 and Corollary 2.8. 
Moreover, in Section 3, we associate to any connected subgraph of $\Pi(L)$ a well defined link type, which we call a molecule of $L$. We prove that the collection (a set with repetitions allowed) of molecules is an isotopy invariant. This collection is a powerful tool for classification questions. In Section 4 we give applications of these concepts to reversibility and chirality questions. Theorem 5.2 is proved in Section 5. The proof uses the action of flypes on the adjacency graph studied in Section 2 and the invariance of molecules proved in Section 3.

Unless otherwise stated, by a link we mean an oriented, alternating, unsplittable, prime link in $S^{3}$, and by a diagram we mean an oriented, alternating, connected, reduced, prime diagram in the 2-sphere $S^{2}$ (not in the plane $R^{2}$ ).

\section{Adjacency}

We recall briefly how atoms are obtained. For more details, see [Quach Hongler and Weber 2004], henceforth abbreviated [QW]. Let $L$ be a link and let $D$ be a diagram representing $L$. We perform a Seifert surgery at each crossing point of $D$ and obtain a bunch $\mathscr{B}$ of disjoint oriented circles in $S^{2}$ called Seifert circles. The site of a surgery is indicated by an arc called the scar of the surgery. If each scar is endowed with a \pm sign, it is easy to reconstruct $D$ from $\mathscr{B}$ and the signed scars. This operation (inverse to surgery) is called suturing the scars. Each circle $\gamma \in \mathscr{B}$ bounds two open discs $\Delta^{\prime}$ and $\Delta^{\prime \prime}$ in $S^{2}$, called the Seifert discs determined by $\gamma$. The set of all Seifert discs determined by the various $\gamma \in \mathscr{B}$ is denoted by $\mathscr{F}$. This set is ordered by inclusion, and a descending chain

$$
\Delta_{0} \supset \Delta_{1} \supset \ldots \supset \Delta_{k}
$$

is said to be of length $k$ and to begin at $\Delta_{0}$.

Definition 2.1. A disc $\Delta \in \mathscr{F}$ is of depth $k$ if

(1) there exists a descending chain of length $k$ beginning at $\Delta$, and

(2) there is no descending chain of length $l$ beginning at $\Delta$ with $l>k$.

According to this definition, innermost discs correspond to discs of depth zero. Following Murasugi [1965], we call a Seifert bunch $\mathscr{B}$ special if each $\gamma \in \mathscr{B}$ bounds an innermost disc.

We now define the Murasugi special components $D^{i}$ (for $i=1,2, \ldots, n$ ) of $D$. First we look at the Seifert discs which are of depth one. Suturing the scars in each such disc, we obtain disjoint, not necessarily prime, special diagrams $D^{1}, \ldots, D^{t}$. Then we remove from each disc of depth one the Seifert discs and scars it contains (we call this operation cleaning). We obtain a new diagram $D^{\prime}$, a new bunch $\mathscr{B}^{\prime}$ and a new set of Seifert discs $\mathscr{F}^{\prime}$. From the Seifert discs of depth one in $\mathscr{F}^{\prime}$, we obtain new special diagrams $D^{t+1}, \ldots, D^{s}$. After cleaning, we obtain a new diagram 
$D^{\prime \prime}$, and so on. Finally the process comes to an end and we obtain the family $D^{1}, \ldots, D^{n}$ of Murasugi special components of $D$.

Although $D$ is supposed to be prime, it may well be that $D^{i}$ is not. To make things clear, recall that a Menasco circle for a diagram $E$ is a Jordan curve $\Gamma$ in $S^{2}$ which cuts $E$ transversally in two points, and such that each disc of $S^{2}$ bounded by $\Gamma$ contains crossing points of $E$. By definition, a diagram is prime if there exists no Menasco circle for it. A beautiful theorem of Menasco [1984] says that if $E$ is prime then the link represented by $E$ is also prime. The reason why there may exist Menasco circles for $D^{i}$ is that $D^{i}$ has been obtained by cleaning many discs, thus allowing the Jordan curve $\Gamma$ to have only two intersection points with $D^{i}$, but many more with $D$.

We define the prime factors $D_{1}^{i}, \ldots, D_{k}^{i}$ of $D^{i}$ to be the subdiagrams of $D^{i}$ which are maximal (with respect to inclusion) and prime. See Figure 1 for an example.

We write $D_{1}, \ldots, D_{s}$ for the diagrams of the prime factors of the various Murasugi components of $D$ (the order is not important). We think of them as being subdiagrams of $D$ and we call them the atom diagrams of $D$.

Definition 2.2. Let $D_{a}$ and $D_{b}$ be two atom diagrams of $D$. We say that they are adjacent if their union $D_{a} \cup D_{b}$ is again a diagram. As this union is necessarily oriented and alternating, the condition is that it is connected and prime.

Lemma 2.3. The two atom diagrams $D_{a}$ and $D_{b}$ are adjacent if and only if:

(1) $D_{a} \cap D_{b}$ consists of exactly one Seifert circle $\gamma \in \mathscr{B}$.

(2) Along $\gamma$ the extremities of the scars of $D_{a}$ are interlaced with those of $D_{b}$.

The proof is easy, once the following definition is stated.

Definition 2.4. Let $X$ and $Y$ be two disjoint, finite subsets of the circle $S^{1}$. We say that they are interlaced if there exist no disjoint intervals $I \supset X$ and $J \supset Y$ in $S^{1}$.

Remark 2.5. Let $\Delta^{\prime}$ and $\Delta^{\prime \prime}$ be the two Seifert discs bounded by $\gamma$. Lemma 2.3 and Definition 2.4 imply that $D_{a} \subset \Delta^{\prime}$ and $D_{b} \subset \Delta^{\prime \prime}$.

Definition 2.6. The adjacency graph $\Pi(D)$ is the graph of the adjacency relation on the set of atom diagrams of $D$.

The graph $\Pi(D)$ is simplicial. Its vertices are labeled by the atom diagrams of $D$ and two vertices are connected by an edge if the corresponding atom diagrams are adjacent. The process is illustrated in Figure 1; in this example the adjacency graph $\Pi(D)$ is

$$
D_{1} \longleftarrow D_{2}-D_{3}-D_{4} \text {. }
$$



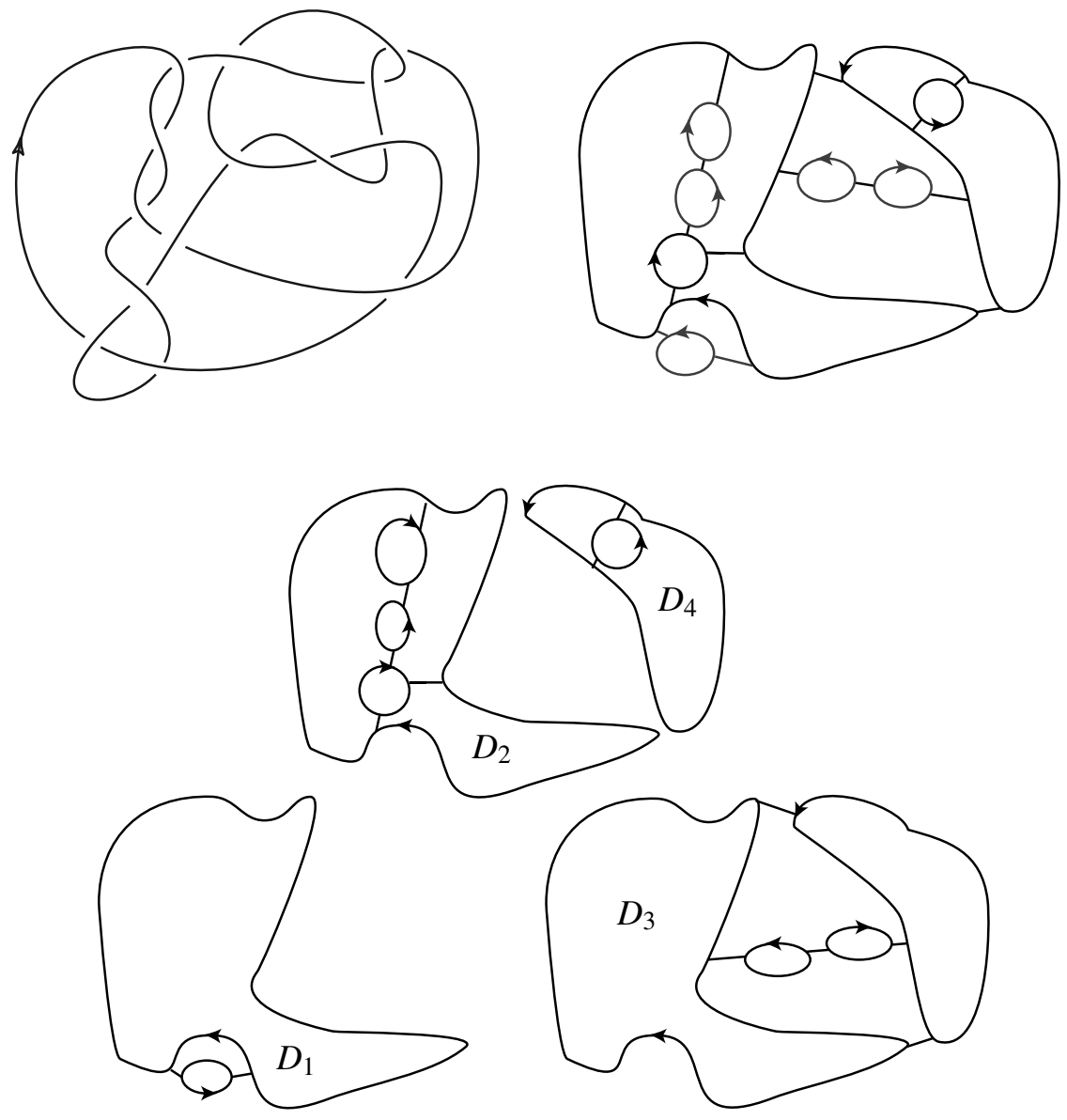

Figure 1. Top left: the initial knot. Top right: the bunch with scars. Bottom: the corresponding atom diagrams.

Theorem 2.7. Let $D$ and $D^{*}$ be two diagrams representing the same link $L$ and suppose that they differ by exactly one flype $f$. Then there exists a canonical isomorphism $\varphi_{f}: \Pi(D) \rightarrow \Pi\left(D^{*}\right)$ such that, for each atom diagram $D_{a}$ of $D$, the atom diagrams $D_{a}$ and $\varphi\left(D_{a}\right)$ represent the same link.

Proof. We shall go back to the proof of the topological invariance of atoms given in $[\mathrm{QW}]$ and extract from it the fact that the adjacency relation is preserved under a flype. Recall that a flype starts from a tangle decomposition like this:

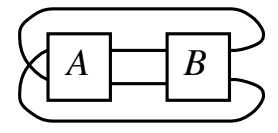


Let $\alpha$ denote the Jordan curve which is the boundary of the tangle $A$.

We ask the following question: How does $\alpha$ cut the Seifert bunch $\mathscr{B}$ associated to the given diagram $D$ ?

The answer is as follows. A Jordan curve $\alpha$ in $S^{2}$ is the boundary of tangle $A$ giving rise to a tangle situation in exactly two cases.

Case 1. (i) $\alpha$ cuts one scar $\sigma$ of $\mathscr{B}$ transversally,

(ii) $\alpha$ cuts transversally twice some Seifert circle $\gamma$ of $\mathscr{B}$ and $\alpha$ does not cut $\mathscr{B}$ somewhere else, and

(iii) $\alpha$ is nontrivial: each disc of $S^{2}$ bounded by $\alpha$ contains some scars distinct from $\sigma$.

A priori there are two possibilities:

(1) No extremity of the the scar $\sigma$ lies on $\gamma$.

(2) One extremity of the scar $\sigma$ lies on $\gamma$.

Recall that the extremities of a scar always lie on different Seifert circles.

Cases 1, 2, and 3 of [QW] correspond to the first possibility, while case 4 corresponds to the second one.

Case 2. The curve $\alpha$ contains a scar $\sigma$ and cuts transversally two Seifert circles of the Seifert bunch $\mathscr{B}$.

Case 5 of $[\mathrm{QW}]$ corresponds to this case.

The analysis carried out in [QW] reveals that, in all five cases examined there, the scar $\sigma$ belongs to some special component, called the supporting component of the flype. This component was denoted $D_{0}$ but will be written $E$ here, for convenience.

We now proceed to analyse the prime components of $E$ and their behaviour under the flype. We shall do this by considering case 1 of [QW], but the arguments are essentially the same in all five cases. We slightly modify Figure 10 of [QW] by sliding $\alpha$ along the scar $\sigma$ to obtain the following:

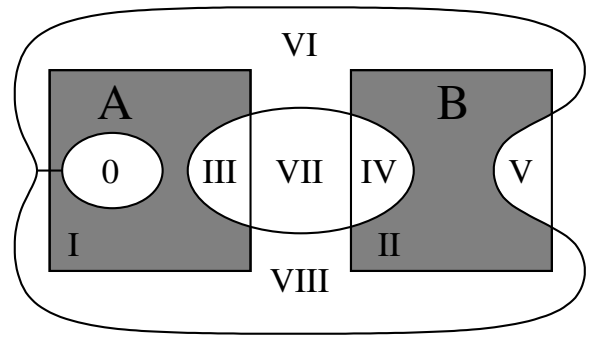

Claim. If $E$ is not prime, Menasco circles for $E$ are entirely contained either in $A$ or in $B$. 
Proof of claim. Think first of the preceding figure before any cleaning has been done. By the definition of a flype, there are no scars outside tangles $A$ and $B$ except for $\sigma$. Hence zones VI, VII and VIII are empty. Zones III, IV and V may each contain some scars and some Seifert circles. These have been removed during the cleaning process which leads to the picture of $E$. Therefore, each of zones III, IV and $\mathrm{V}$ may contain atom diagrams which are adjacent to a prime factor of $E$. We now look at zones I and II, as sketched in the figure. Both zones must contain discs and scars of $E$. This is because zone 0 and zone III must be connected through zone I by scars and discs of $E$, or else the crossing of $D$ which corresponds to the scar $\sigma$ would be nugatory. For the same reason, zones IV and V must be connected through zone II.

We deduce from the above argument that the prime factors of $E$ (namely the atom diagrams produced by $E$ ) are as follows:

(1) The atom diagram $E_{0}$ which contains the scar $\sigma$, the two Seifert circles to which $\sigma$ is attached, the Seifert circle which is the boundary of (zone III $U$ zone IV $\cup$ zone VII), and some of the boundaries of colored discs and scars which are in zone I and in zone II.

(2) Maybe some other prime factors, each of them entirely contained in tangle $A$ or in tangle $B$. Denote these possible atom diagrams by $E_{i}$ for $i=1, \ldots, t$.

We look again at the figure on the previous page and search for the atom diagrams which are adjacent to $E_{0}$ or to some $E_{i}$ for $i=1, \ldots, t$. To do this, we consider the initial bunch $\mathscr{B}$ and we perform cleaning operations in the coloured discs of depth 1 or greater, until they become exactly of depth 1 . We thus obtain a bunch $\mathscr{R}^{\prime}$ and a corresponding diagram $D^{\prime}$. We write $G$ for an atom diagram of $D^{\prime}$ which is contained in a coloured disc.

Question. Which are the $G$ s adjacent to $E_{0}$ ?

Answer. The $G$ s which are adjacent to $E_{0}$ are:

(i) Those contained in zone 0 , zone III, zone IV or zone V.

(ii) Some of the $G$ s contained in the coloured discs situated in $A$ or $B$.

Question. Where are the $G$ 's adjacent to some $E_{i}$ for $i=1, \ldots, t$ ?

Answer. (iii) They are in some coloured disc situated in $A$ or in $B$.

What makes the difference between (ii) and (iii) depends on the position of the atom diagram $G$ with respect to the Menasco circles for $E$. For each such Menasco circle $\mu_{j}$ consider the disc $M_{j}$ bounded by $\mu_{j}$ in tangle $A$ or in tangle $B$. Now $G$ is adjacent to $E_{0}$ if it is outside all the $M_{j}$ and it is adjacent to some $E_{i}$ if it is inside some $M_{j}$. 
We now consider the situation after the flype. The new diagram is $D^{*}$ with its corresponding bunch $\mathscr{B}^{*}$. The supporting diagram is now the Murasugi special component of $D^{*}$ which contains the scar $\sigma^{*}$. In [QW] we proved that this supporting component is the image by the flype of the supporting component of $D$. This is the equality $\left(D^{*}\right)_{0}=\left(D_{0}\right)^{*}$ of $[\mathrm{QW}]$. We write here $E^{*}$ for the new supporting component. The situation after the flype is this:

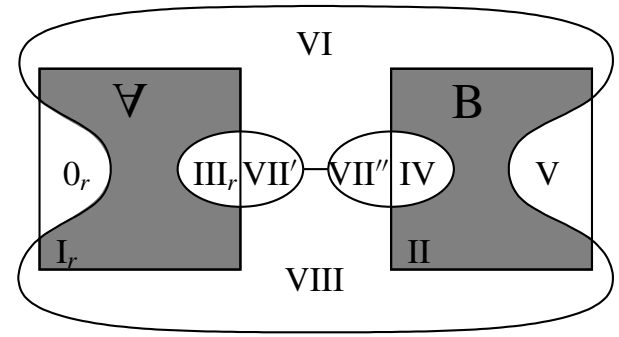

We see that:

(1) Zone VII has disappeared, giving birth to zones VII' and VII". This is of no consequence because they are empty.

(2) The scar $\sigma$ has, of course, disappeared. Zone 0 has undergone a 180-degree rotation and been connected to the big Seifert disc which contains the point at infinity.

(3) Zones I and II have been turned.

We define $\left(E^{*}\right)_{0}$ to be the prime factor of $E^{*}$ which contains the scar $\sigma^{*}$.

Claim. The equality $\left(E^{*}\right)_{0}=\left(E_{0}\right)^{*}$ holds, which allows us to write simply $E_{0}{ }^{*}$.

Proof of claim. The equality can be seen by comparing the two preceding figures. Here are some details. In the figure above, $E_{0}{ }^{*}$ is the atom diagram which contains

(a) the huge circle bounding the Seifert disc which contains the point at infinity,

(b) the boundary of (turned zone III) $\cup$ zone VII',

(c) the boundary of zone IV $\cup$ zone VII',

(d) the part of $E_{0}$ which is in zone II, and

(e) the part of $E_{0}$ which was in zone I, turned by a 180 degree rotation.

The key points from which the equality $\left(E^{*}\right)_{0}=\left(E_{0}\right)^{*}$ can be deduced are:

(1) The Menasco circles which were in tangle $B$ before the flype have not been moved.

(2) The Menasco circles which were in tangle $A$ before the flype have simply been turned. 
We now construct the promised isomorphism

$$
\varphi: \Pi(D) \rightarrow \Pi\left(D^{*}\right)
$$

We define the image of $E_{0}$ by $\varphi$ to be $E_{0}{ }^{*}$. As they differ by a flype, they represent the same atom.

Any other atom diagram of $D$ is entirely contained in either tangle $A$ or in tangle $B$. If it is contained in $B$, then $\varphi$ sends it to itself. If it is in $A$, then $\varphi$ sends it to its image under the rotation. From this, we immediately deduce that if two adjacent atom diagrams of $D$ are entirely contained in $A$ or in $B$, the adjacency relation is preserved by $\varphi$.

It remains to see what happens to atom diagrams of $D$ which are adjacent to $E_{0}$. For those which are contained in $B$ (that is, in zones II, IV and V) their image under $\varphi$ will be adjacent to $E_{0}{ }^{*}$ because nothing changes in $B$ under the flype. Those which are contained in $A$ are turned by the flype. For those in zones 0 and III, their image will clearly be adjacent to $E_{0}{ }^{*}$. This is also true for those in zone I, because their image under the rotation lies outside the image of all the Menasco circles. This completes the proof of Theorem 2.7.

Corollary 2.8. Let $D$ and $D^{\prime}$ be two diagrams representing the same link type $L$. Then $\Pi(D)$ and $\Pi\left(D^{\prime}\right)$ are isomorphic.

Proof. The main result of [Menasco and Thistlethwaite 1993] asserts that $D$ and $D^{\prime}$ differ by a finite sequence of flypes and homeomorphisms of $S^{2}$.

Remark. Corollary 2.8 says that there exists an isomorphism $\Psi$ between $\Pi(D)$ and $\Pi\left(D^{\prime}\right)$ such that, for each vertex $D_{a}$ of $\Pi(D)$, the atom diagrams $D_{a}$ and $\Psi\left(D_{a}\right)$ represent isotopic links. It would be pleasing to have a canonical isomorphism between the two adjacency graphs, but in general this is not possible because diagrams can have nontrivial automorphisms. To amend this state of affairs, we can categorize. Here are some brief details.

Let $\Gamma$ and $\Gamma^{\prime}$ be two graphs embedded in $S^{2}$. A map $\Phi:\left(S^{2}, \Gamma\right) \rightarrow\left(S^{2}, \Gamma^{\prime}\right)$ is a homeomorphism $\Phi: S^{2} \rightarrow S^{2}$ of degree +1 such that $\Phi(\Gamma)=\Gamma^{\prime}$. An isotopy is a continuous family $\Phi_{t}$ of maps $\left(S^{2}, \Gamma\right) \rightarrow\left(S^{2}, \Gamma^{\prime}\right)$ for $t \in[0,1]$. An isotopy class of maps will be called a spherical equivalence.

The difficulty about the non-canonicity of $\Psi$ is that the group of spherical equivalences of a diagram in $S^{2}$ can be nontrivial. The following easily proved lemma will help.

Lemma 2.9. Let $\Gamma$ be a connected graph in $S^{2}$ and let e be an oriented edge of $\Gamma$. Then a spherical equivalence $\Phi:\left(S^{2}, \Gamma\right) \rightarrow\left(S^{2}, \Gamma\right)$ such that $\Phi(e)=e$ keeping the orientation of e fixed, is the identity.

As an application of the lemma, consider a diagram $D$ and its image $D^{*}$ by a flype. Now, $D^{*}$ is actually not a well defined graph embedded as a subspace of $S^{2}$, 
as several choices are involved. But any two representatives for $D^{*}$ are canonically isomorphic, because a flype is the identity on some part of $D$.

With this in hand, we can consider the category whose objects are diagrams in $S^{2}$ and whose morphisms are flypes. Then the adjacency graph is a functor $\Pi$ from this category to the category of simplicial graphs, with labeled vertices. The labels are the atom diagrams. This is a sophisticated way to state that the adjacency graph is an isotopy invariant.

However, we shall often dare to speak of the adjacency graph $\Pi(L)$ of a link $L$. The vertices of $\Pi(L)$ are then labeled by the atoms of $L$.

Proposition 2.10. The adjacency graph $\Pi(L)$ of a link $L$ is bipartite: adjacent vertices represent prime special links of opposite sign.

Proof. Let $D$ be a special diagram. Then all crossings of $D$ have the same Conway sign. We have seen above (see Remark 2.5) that if $D_{a}$ and $D_{b}$ are two adjacent atom diagrams, there exists a Seifert circle $\gamma$ such that $D_{a} \cap D_{b}=\gamma$. If $\Delta^{\prime}$ and $\Delta^{\prime \prime}$ are the two Seifert discs bounded by $\gamma$, then $D_{a} \subset \bar{\Delta}^{\prime}$ and $D_{b} \subset \bar{\Delta}^{\prime \prime}$. As $D_{a} \cup D_{b}$ is alternating, this implies that the sign of $D_{a}$ is the opposite of the sign of $D_{b}$.

Now, the sign of a special diagram is preserved by a flype. Hence the isomorphism $\varphi_{f}: \Pi(D) \rightarrow \Pi\left(D^{\prime}\right)$, associated to a flype $f$, preserves the signs of the vertices. As a consequence, atoms have signs and two atoms which are the extremities of an edge have opposite signs.

We wish to produce some examples. This raises a problem of notation, because the knots pictured in the tables are not oriented. This is not too consequential for the examples given below, because the knots we shall use have few crossings and hence are usually reversible. The situation is worse for links. We shall deal more extensively with these matters in Section 4. We write the sign as an exponent, hence $3{ }_{1}{ }^{+}$denotes a trefoil with positive Conway signs.

Example 2.11. The knots $10_{42}$ and $10_{43}$ have the same collection

$$
\left\{2_{1}^{2^{+}}, 2_{1}^{2^{-}}, 3_{1}^{+}, 3_{1}^{-}\right\}
$$

of atoms. Their unlabeled adjacency graph is

$$
A-B-C \longrightarrow D
$$

in both cases. The vertices are labeled

$$
A=3_{1}^{+}, \quad B=3_{1}^{-}, \quad C=2_{1}^{2^{+}}, \quad D=2_{1}^{2-}
$$

for the knot $10_{42}$, and

$$
A=3_{1}^{+}, \quad B=2_{1}^{2^{-}}, \quad C=2_{1}^{2^{+}}, \quad D=3_{1}^{-}
$$

for the knot $10_{43}$. 
We see that the graphs with vertices labeled by the atoms are non-isomorphic. Incidentally, this shows that the two knots are distinct.

Not surprisingly, the adjacency graph is too weak to distinguish knots. For instance, the knots $10_{115}$ and $10_{43}$ have the same adjacency graph. The smallest such examples are provided by the graph $3_{1}^{+}-3_{1}^{-}$which corresponds to the knot $6_{3}$ but also to the link $6_{2}^{3}$ and by the graph $2_{1}^{2^{+}-}-4_{1}^{2^{-}}$which corresponds to the knot $6_{2}$ and to the link $6_{1}^{3}$ for some orientation.

\section{Molecules}

Proposition 3.1. Let $L$ be a link and let $D$ and $D^{\prime}$ be two diagrams for $L$ differing by a flype $f$. Let $H \subset \Pi(D)$ be a connected subgraph of $\Pi(D)$ and let $L(H)$ be the link represented by the union of the atom diagrams which correspond to the vertices of $H$. Let $\varphi_{f}: \Pi(D) \rightarrow \Pi\left(D^{\prime}\right)$ be the canonical isomorphism induced by the flype $f$. Then $L(H)$ and $L(\varphi(H))$ represent the same link type.

As a consequence, again thanks to the Menasco-Thistlethwaite Flyping Theorem, if $L$ is a given link, we can associate to any connected subgraph of $\Pi(L)$ a well defined link type. The collection of links obtained this way from the set of connected subgraphs of $\Pi(L)$ will be called the collection of molecules of $L$ and we shall denote it by $M(L)$. As for the collection of atoms, we remark that a given link type may appear several times in the list. Notice that molecules are prime links, as they correspond to connected adjacency subgraphs.

Warning. If we want to point out to a particular molecule, "the" graph $\Pi(L)$ may be too imprecise. This is especially the case when "the" adjacency graph has nontrivial automorphisms (as a labeled graph). Then it is better to use the more elaborate categorical setting sketched above.

Example 3.2. A necessary condition for two links $L$ and $L^{\prime}$ to be isotopic is that $M(L)=M\left(L^{\prime}\right)$. Consider the knots $10_{45}$ and $10_{88}$. Both have the same adjacency graph (and hence the same atoms)

$$
2_{1}^{2^{+}}-3_{1}^{-}-3_{1}^{+}-2_{1}^{2^{-}} \text {. }
$$

However the collection of molecules distinguishes the two knots, because the molecule associated to the subgraph $3_{1}^{-}-3_{1}^{+}$is the knot $6_{3}$ for $10_{45}$ and "the" link $6_{2}^{3}$ for $10_{88}$.

Proof of Proposition 3.1. The detailed proof of Theorem 2.7 contains all the ingredients necessary to prove Proposition 3.1. Two possibilities can occur:

If $H$ contains a prime factor of the supporting diagram of the flype $f$, then $L(\varphi(H))$ is represented by $H^{*}$, the image of $H$ under the flype. 
If $H$ does not contain a prime factor of the supporting diagram, then its image is translated and/or turned.

We now consider the molecules which are built up from two adjacent atom diagrams. In fact, the existence of an edge between two vertices says that some bond exists between the two corresponding atoms. The edge in itself says nothing about the nature of the bond, but the two-atom molecule reveals what this bond is. We call such molecules edge-molecules.

One can then add a weight on the edges of the adjacency graph, the weight being the edge-molecules; we call the resulting graph the bond graph. It is very tempting to conjecture that a link is determined by its bond-graph, but this is (alas?) wrong. An example is provided by the graph

$$
2_{1}^{2+}-3_{1}^{-}-2_{1}^{2+} \text {. }
$$

The edge-molecules are the knot $5_{2}$. But the knot $7_{7}$ and the link $7_{1}^{3}$ (for some orientation) both have this weighted graph as bond graph.

Another temptation is to look at the vertex stars. By definition, if $P$ is a vertex in a simplicial graph $\Pi$, the $\operatorname{star} \operatorname{st}(P)$ is the subgraph of $\Pi$ which is the union of the edges (and their extremities) which have $P$ as a vertex. Accordingly, if $L_{i}$ is the atom which corresponds to a vertex of $\Pi(L)$ the molecule associated to the star $\operatorname{st}\left(L_{i}\right)$ describe a kind of neighborhood of $L_{i}$ in $L$.

Question. Let $L$ and $L^{\prime}$ be two links and suppose that there exists an isomorphism $\varphi: \Pi(L) \rightarrow \Pi\left(L^{\prime}\right)$ such that, for any atom $L_{i}$ of $L, \operatorname{st}\left(L_{i}\right)$ and $\operatorname{st}\left(\varphi\left(L_{i}\right)\right)$ are isotopic. Are $L$ and $L_{i}$ isotopic?

The answer is, in general, no (mutations can be used to construct counterexamples) but the answer might be yes if the graph is 2-connected.

\section{Applications to chirality and reversibility}

If $L$ is a link, we denote by $\hat{L}$ the mirror image of $L$ as an oriented link. We denote by $-L$ the oriented link obtained by changing the orientation of all the components of $L$. The same notations will be used for diagrams.

If $\Pi(L)$ is the adjacency graph of $L$, the graph obtained from $\Pi(L)$ by replacing the label $L_{i}$ at each vertex by the label $\hat{L}_{i}$ will be written $\widehat{\Pi}(L)$. Clearly $\Pi(\hat{L})=$ $\widehat{\Pi}(L)$. Analogously $-\Pi(L)$ denotes the adjacency graph obtained from $\Pi(L)$ by replacing each vertex label $L_{i}$ by $-L_{i}$. Obviously one has $-\Pi(L)=\Pi(-L)$. We recall now the usual definitions.

Definition 4.1. A link $L$ is positively achiral if it is isotopic to $\hat{L}$, negatively achiral if it is isotopic to $-\hat{L}$, and reversible if it is isotopic to $-L$. 
Theorem 4.2. If the link $L$ is positively achiral, the graph $\Pi(L)$ is isomorphic to $\widehat{\Pi}(L)$. If it is negatively achiral, the graph $\Pi(L)$ is isomorphic to $-\widehat{\Pi}(L)$. If it is reversible, the graph $\Pi(L)$ is isomorphic to $-\Pi(L)$.

Proof. The proof is an immediate consequence of the isotopy invariance of the adjacency graph, loosely stated.

We write $\widehat{M}(L)$ for the collection of molecules of the link $\hat{L}$. Analogously, we write $-M(L)$ and $-\widehat{M}(L)$ for the molecule collection of, respectively, $-L$ and $-\hat{L}$.

Corollary 4.3. (1) If $L$ is positively achiral, then $M(L)=\widehat{M}(L)$.

(2) If $L$ is negatively achiral, then $\mu(L)=-\widehat{M}(L)$.

(3) If $L$ is reversible, one has $M(L)=-M(L)$.

We now produce some examples. As far as chirality questions are concerned, it is easy to produce many, because atoms are chiral. For instance, consider the knot $10_{42}$ whose adjacency graph

$$
3_{1}^{+}-3_{1}^{-}-2_{1}^{2+}-2_{1}^{2^{-}}
$$

was introduced in Example 2.11. We see that the atoms can be grouped in pairs $(D, \widehat{D})$ so that the knot passes the first test for achirality. However, the graph $\Pi(D)$ is not isomorphic to $\widehat{\Pi}(D)$ and hence $10_{42}$ is chiral.

Reversibility questions are more delicate to handle, because atoms can be reversible or non-reversible. In each case, an ad hoc proof is needed. Here is an example of a diagram $D$ for a 3-component link:

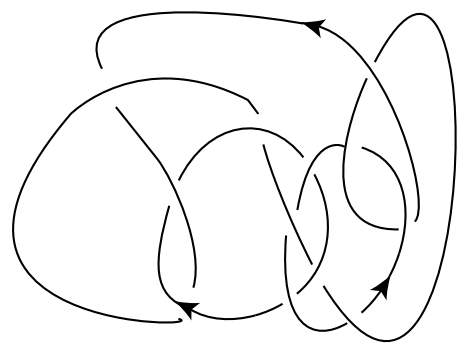

The adjacency graph $\Pi(D)$ is<smiles>[3H][14CH2][Te][3H]</smiles>

The edge molecule associated to the middle bond is the knot $8_{17}$ which is known to be non-reversible. This implies that the link is also non-reversible. 


\section{Decomposition of achiral links as a Murasugi sum}

Definition 5.1. Let $L$ be a link and $\left\{M_{1}, \ldots, M_{s}\right\}$ be a collection of molecules whose atoms constitute a partition of the collection of atoms of $L$. Let $D$ be a diagram for $L$ and let $D_{1}, \ldots, D_{s}$ be the subdiagrams of $D$ representing the molecules $M_{1}, \ldots, M_{s}$. Suppose that all the $D_{i}$ have the same Seifert circle $\gamma$ in common. Then we say that the molecule diagrams $D_{1}, \ldots, D_{s}$ form a decomposition of $D$ as a diagrammatic Murasugi sum.

The pioneering work of Murasugi shows that the link $L$ is a Murasugi sum, in the classical sense, of the links $M_{1}, \ldots, M_{s}$. The Seifert circle $\gamma$ will be called a plumbing (Seifert) circle. Traditionally one considers Murasugi sums with two factors, and this can easily be achieved at the cost of dealing with possibly nonprime link factors. For instance, one can choose an integer $k$ such that $1<k<s$ and consider the subdiagrams $D^{\prime}=\bigcup_{i=1, \ldots, k} D_{i}$ and $D^{\prime \prime}=\bigcup_{i=(k+1), \ldots, s} D_{i}$. If $L^{\prime}$ is the link represented by $D^{\prime}$ and if $L^{\prime \prime}$ is the link represented by $D^{\prime \prime}$, then $L$ is a Murasugi sum $L=L^{\prime} * L^{\prime \prime}$ with two factors.

The aim of this section is to prove the following theorem.

Theorem 5.2. (1) If $L$ is positively achiral, there exists a link $L^{\prime}$ such that $L=$ $L^{\prime} * \hat{L}^{\prime}$.

(2) If $L$ is negatively achiral, there exists a link $L^{\prime}$ such that $L=L^{\prime} *-\hat{L}^{\prime}$.

Remark. The decomposition is not necessarily unique and the link $L^{\prime}$ is not necessarily prime. How this can happen is explained below; see the proof of Theorem 5.2 when $\Pi(D)$ is 2 -connected.

Proof. We prove the theorem when $L$ is positively achiral. If $L$ is negatively achiral, it suffices to replace $\widehat{\Pi}(D)$ by $-\widehat{\Pi}(D)$ in the proof given below.

Let $D$ be a diagram for $L$. We consider the graphs $\Pi(D)$ and $\widehat{\Pi}(D)$. By [Menasco and Thistlethwaite 1993] and Theorem 2.7 there exists a sequence of flypes which induces an isomorphism from $\widehat{\Pi}(D)$ to $\Pi(D)$. As such a sequence is not necessarily unique, we choose one and write

$$
\Phi: \widehat{\Pi}(D) \rightarrow \Pi(D)
$$

for the induced isomorphism.

We now write $\breve{\Pi}(D)$ for the graphs $\Pi(D)$ or $\widehat{\Pi}(D)$ with no label at the vertices. Then $\Phi$ induces an isomorphism of (plain) graphs

$$
\breve{\Phi}: \breve{\Pi}(D) \rightarrow \breve{\Pi}(D) \text {. }
$$

Remark 5.3. No vertex of $\breve{\Pi}(D)$ is fixed by $\breve{\Phi}$, as $\Phi$ sends a vertex of $\widehat{\Pi}(D)$ to a vertex of $\Pi(D)$ with the same label. As a consequence, no edge of $\breve{\Pi}(D)$ is pointwise fixed by $\breve{\Phi}$. 
Proof of Theorem 5.2 when $\breve{\Pi}(D)$ is a tree. By the Hopf-Lefschetz fixed point theorem, $\breve{\Phi}$ has fixed points. Remark 5.3 implies that $\breve{\Phi}$ has a unique fixed point, and that this fixed point is located in the middle of an edge $e$. Now remove the interior of $e$. Then $\Pi(D)$ is split into two subtrees $\Pi(D)^{\prime}$ and $\Pi(D)^{\prime \prime}$. Similarly, $\widehat{\Pi}(D)$ is split into $\widehat{\Pi}(D)^{\prime}$ and $\widehat{\Pi}(D)^{\prime \prime}$. As $\breve{\Phi}$ exchanges the extremities of the edge $e, \Phi$ induces an isomorphism of $\widehat{\Pi}(D)^{\prime}$ onto $\Pi(D)^{\prime \prime}$, and of $\widehat{\Pi}(D)^{\prime \prime}$ onto $\Pi(D)^{\prime}$. The topological invariance of molecules proved in Proposition 3.1 implies that the molecule $L^{\prime}$ associated to $\Pi(D)^{\prime}$ is the mirror image $\hat{L}^{\prime}$ of the molecule associated to $\Pi(D)^{\prime \prime}$.

On the other hand, the edge $e$ has two vertices $V^{\prime} \in \Pi(D)^{\prime}$ and $V^{\prime \prime} \in \Pi(D)^{\prime \prime}$. The edge between $V^{\prime}$ and $V^{\prime \prime}$ indicates a Murasugi sum between the atom represented by $V^{\prime}$ and the atom represented by $V^{\prime \prime}$. Indeed, the adjacency relation represented by $e$ says precisely that the two atoms have a common Seifert circle $\gamma$. But this argument also shows that we have a diagrammatic Murasugi sum between $\Pi(D)^{\prime}$ and $\Pi(D)^{\prime \prime}$. As a consequence, $\mathrm{L}$ is a Murasugi sum of the molecule $L^{\prime}$ and the molecule $\hat{L}^{\prime}$.

In order to be able to argue when $\Pi(D)$ is not a tree, we need some facts about simplicial graph theory. A general reference for the results we need is provided by the book [Diestel 2000]. A circuit is a graph which is homeomorphic to a circle. A graph $G$ is 2-connected if it contains at least three vertices and if any two distinct vertices are situated on at least one circuit. By Menger's theorem, a connected $G$ is 2-connected if and only if it contains no cut vertex and is not the connected graph with only one vertex or only one edge.

Proposition 5.4. Suppose that $\breve{\Pi}(D)$ is 2-connected. Then all atom diagrams of $D$ have one Seifert circle $\gamma$ in common.

Proof of Proposition 5.4. For the moment, let $D$ be any diagram (not necessarily prime) and let $\gamma$ be a Seifert circle of $D$. Let $\Delta^{\prime}$ and $\Delta^{\prime \prime}$ be the two open discs of $S^{2}$ bounded by $\gamma$, and let $\bar{\Delta}^{\prime}$ and $\bar{\Delta}^{\prime \prime}$ be their closure. We define the depth $d_{\gamma}$ of $\gamma$ to be the pair of integers $\left(k_{\gamma}^{\prime}, k_{\gamma}^{\prime \prime}\right)$ where $k_{\gamma}^{\prime}$ is the depth of $\Delta^{\prime}$ and $k_{\gamma}^{\prime \prime}$ the one of $\Delta^{\prime \prime}$. After a possible change of notation we can assume that $0 \leq k_{\gamma}^{\prime} \leq k_{\gamma}^{\prime \prime}$.

Remark. If $k_{\gamma}^{\prime}=0$ for all $\gamma$ in the bunch $\mathscr{B}$, then $k_{\gamma}^{\prime \prime}=1$ for all $\gamma \in \mathscr{B}$ and, in fact, the diagram $D$ is special. In this case, the graph $\Pi(D)$ has no edge. It is a disjoint union of vertices, one vertex for each atom diagram. However, if there are more than one atom present, one can consider that $D$ is a diagrammatic Murasugi sum, as it is a connected sum.

As a consequence, if $\Pi(D)$ is 2-connected, there must exist Seifert circles $\gamma$ with $k_{\gamma}^{\prime} \geq 1$.

Claim. If $\Pi(D)$ is 2-connected, and if $\gamma \in \mathscr{B}$ such that $k_{\gamma}^{\prime} \geq 1$ then $k_{\gamma}^{\prime}=k_{\gamma}^{\prime \prime}=1$. 
Proof of claim. Suppose that $k_{\gamma}^{\prime \prime} \geq 2$. Choose a descending sequence

$$
\Delta_{\gamma}^{\prime \prime} \supset \Delta_{1} \supset \cdots \supset \Delta_{n}
$$

of Seifert discs, with $n \geq 2$. Let $\gamma_{i}$ be the boundary of $\Delta_{i}$. Consider the unique atom diagram $D_{0}$ which has $\gamma$ and $\gamma_{1}$ as Seifert circles. (It is easy to see that given any two Seifert circles of a Seifert bunch, there is at most one atom diagram which contains these two circles.) As $k_{\gamma}^{\prime} \geq 1$, there are atom diagrams in $\bar{\Delta}^{\prime}$, and by construction there are atom diagrams in $\bar{\Delta}_{1}$. As $D_{0}$ is unique, if one removes the vertex $D_{0}$ from the graph $\Pi(D)$, there is no way to connect, in the remaining graph, atom diagrams in $\bar{\Delta}^{\prime}$ to atom diagrams in $\bar{\Delta}_{1}$. In other words, $D_{0}$ is a cutvertex of $\Pi(D)$, which contradicts the assumption that $\Pi(D)$ is 2-connected.

Now we observe that, in any connected diagram, there cannot exist more than one Seifert circle $\gamma$ with $d_{\gamma}=(1,1)$. Let $\gamma$ be the unique such Seifert circle for $D$. All atom diagrams contain $\gamma$. This completes the proof of Proposition 5.4.

Proof of Theorem 5.2 when $\breve{\Pi}(D)$ is 2-connected. We know from Proposition 5.4 that there exists exactly one Seifert circle $\gamma$ with $d_{\gamma}=(1,1)$. We claim that $\gamma$ is the plumbing circle we seek. Let $\Delta^{\prime}$ and $\Delta^{\prime \prime}$ be the two open discs bounded by $\gamma$ in $S^{2}$. Let $D_{1}{ }^{\prime}, \ldots, D_{s}{ }^{\prime}$ be the atom diagrams which are in $\bar{\Delta}^{\prime}$ and have $\gamma$ as one of their Seifert circles, and let $D_{1}{ }^{\prime \prime}, \ldots, D_{t}^{\prime \prime}$ be those which are in $\bar{\Delta}^{\prime \prime}$. In fact, there are no more atoms in $D$, otherwise $\gamma$ would not be of depth $(1,1)$. The atoms in $\bar{\Delta}^{\prime}$ have all the same sign $\left(\right.$ say + ) and those in $\bar{\Delta}^{\prime \prime}$ have all the opposite sign (say - ).

Note. This argument shows that every 2-connected adjacency graph is a subgraph of the complete bipartite graph on $(s, t)$ vertices. We do not know which subgraphs can actually be obtained this way. It is easy to realize the complete graphs.

Now, the isomorphism $\Phi$ exchanges the atoms which are in $\bar{\Delta}^{\prime}$ with those which are in $\bar{\Delta}^{\prime \prime}$, and hence $s=t$. Let $L_{i}^{\prime}$ be the link represented by $D_{i}^{\prime}$ and, similarly, let $L_{j}^{\prime \prime}$ be the link represented by $D_{j}^{\prime \prime}$, for $i=1, \ldots, s$ and $j=1, \ldots, s$. After a possible change of numbering we can assume that $\Phi\left(\widehat{D}_{i}^{\prime}\right)=D_{i}^{\prime \prime}$ for $i=1, \ldots, s$. As $\Phi$ is induced by flypes, $\hat{L}_{i}^{\prime}$ is isotopic to $L_{i}^{\prime \prime}$ for $i=1, \ldots, s$. Write $L^{\prime}$ for the connected sum of the $L_{i}^{\prime}$ for $i=1, \ldots, s$, and $L^{\prime \prime}$ for the connected sum of the $L_{i}^{\prime \prime}$ for $i=1, \ldots s$. Then $\hat{L}^{\prime}$ is isotopic to $L^{\prime \prime}$ and $L$ is a Murasugi sum of $L^{\prime}$ and $L^{\prime \prime}$.

We now need a slight modification of the block decomposition of a graph. To avoid confusion we shall use the word "brick".

Definition 5.5. A brick of a connected graph $G$ is a subgraph of $G$ which is 2connected and maximal (with respect to inclusion) for this property.

Let $\left\{B_{1}, \ldots, B_{s}\right\}$ be the bricks of the connected graph $G$. If $a \neq b$ then $B_{a} \cap B_{b}$ is either empty or consists of just one vertex. Let 


$$
A=G \backslash \bigcup_{i=1}^{s} \operatorname{Int}\left(B_{i}\right)
$$

$A$ is a forest, that is, a disjoint union of trees. Let

$$
A=\coprod_{j=1}^{t} A_{j}
$$

where each $A_{j}$ is a tree. We now perform the following enlargement on $G$.

Let $B_{a}$ be a brick. Then the intersection $B_{a} \cap A$ is a finite set of vertices $\left\{P_{1}, \ldots, P_{u}\right\}$. Consider the disjoint union

$$
\left(G \backslash \operatorname{Int} B_{a}\right) \amalg B_{a} .
$$

Each vertex $P_{1}, \ldots, P_{u}$ appears once in $\left(G \backslash \operatorname{Int} B_{a}\right)$ and once in $B_{a}$. From the disjoint union we construct a new graph by joining both appearances of $P_{i}$ by a new edge for $i=1, \ldots, u$. We perform the same operation for each brick $B_{a}$. At the end, we get a connected graph $\breve{G}$. Here is an example:
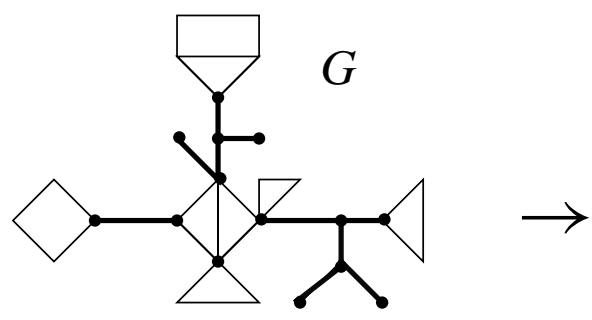

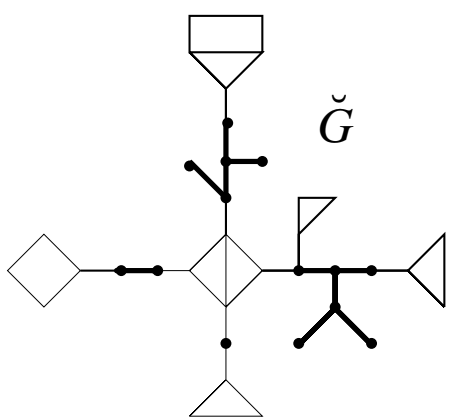

$\check{G}$ contains canonically each brick $B_{i}$ and each tree $A_{j}$. In $\check{G}$ one has $B_{i} \cap B_{k}=\varnothing$ if $i \neq k$ and $A_{j} \cap B_{i}=\varnothing$ for all $i=1, \ldots, s$ and all $j=1, \ldots, t$. In $\check{G}$ we now contract separately to a point each brick $B_{i}$. This quotient graph is written $\bar{G}$ :

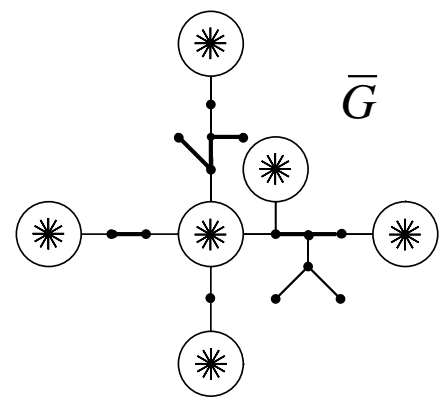


It is easy to prove that $\bar{G}$ is a tree. This tree has two kinds of vertices. Firstly, the vertices of the forest $A$, which we call ancient vertices. Secondly, the vertices which correspond to the smashed bricks, denoted by a star on the figure above, and which we call brick vertices.

Suppose now that $\Psi: G \rightarrow G$ is a graph automorphism. The construction of $\check{G}$ and of $\bar{G}$ is so natural that $\Psi$ induces automorphisms $\check{\Psi}: \check{G} \rightarrow \check{G}$ and $\bar{\Psi}: \bar{G} \rightarrow \bar{G}$. The automorphism $\bar{\Psi}$ sends a brick vertex to a brick vertex and an ancient vertex to an ancient one.

Proof of Theorem 5.2 in the general case. We apply the bar construction above to the graph $G=\breve{\Pi}(D)$. We write $\bar{\Pi}(D)$ for the graph thus obtained from $\breve{\Pi}(D)$. As $\bar{\Pi}(D)$ is a tree, the automorphism $\bar{\Phi}$ has fixed points.

Claim. $\bar{\Phi}$ has exactly one fixed point. It is either the mid-point of an edge whose extremities are two ancient vertices or a brick vertex.

Proof of claim. By construction, the vertices of an edge of $\bar{\Pi}(D)$ are either two ancient vertices or one brick vertex and one ancient vertex. As every ancient vertex is moved by $\bar{\Phi}$, no edge is pointwise fixed by $\bar{\Phi}$. This implies that $\bar{\Phi}$ has exactly one fixed point and that this fixed point is of one of the two forms as stated.

Suppose that the fixed point is the mid-point of an edge $\bar{e}$. Let $e$ be the edge of $\breve{\Pi}(D)$ which projects onto $\bar{e}$. This edge $e$ is a bridge of $\breve{\Pi}(D)$. The automorphism $\breve{\Phi}: \breve{\Pi}(D) \rightarrow \breve{\Pi}(D)$ has the mid-point of $e$ as fixed point. From here, the proof proceeds in the same way as when $\breve{\Pi}(D)$ is a tree. We remove the interior of $e$ from $\breve{\Pi}(D)$ which is thus split into two connected subgraphs permuted by $\breve{\Phi}$ and so on.

Suppose that the fixed point is a brick vertex. By construction, this means that there is a brick $B_{a}$ which is invariant by $\breve{\Phi}$. Remove the fixed point from $\bar{\Pi}(D)$. The remaining graph is a disjoint union of trees and these trees are permuted by $\bar{\Phi}$. No tree is invariant, as there is only one fixed point. Hence, we can find a partition $\left\{A_{1}, \bar{\Phi}\left(A_{1}\right), \ldots, A_{n}, \bar{\Phi}\left(A_{n}\right)\right\}$ of the set of trees. Now consider the graph $\breve{\Pi}(D)$ and remove from it the interior of the brick $B_{a}$. The remaining graph is a disjoint union of subgraphs. We write $G_{j}$ for the subgraph which projects onto $A_{j}$ (for $j=1, \ldots, n$ ). The remaining subgraph is indeed the disjoint union of $\left\{G_{1}, \breve{\Phi}\left(G_{1}\right), \ldots, G_{n}, \breve{\Phi}\left(G_{n}\right)\right\}$. The intersection $G_{j} \cap B_{a}$ is a cut vertex $P_{j}$ of $\breve{\Pi}(D)$.

We return to the proof of Theorem 5.2 when $\breve{\Pi}(D)$ is 2-connected. Consider the brick $B_{a}$ of $\breve{\Pi}(D)$. Let $\gamma$ be the plumbing circle for $B_{a}$. Let $\Delta^{\prime}$ and $\Delta^{\prime \prime}$ be the Seifert discs bounded by $\gamma$ as above. After a possible change of notation, we can assume that the diagram $D_{j}$ which corresponds to $G_{j}$ is contained in $\Delta^{\prime}$ for $j=1, \ldots, n$. Hence, the diagram which corresponds to $\breve{\Phi}\left(G_{j}\right)$ is contained in $\Delta^{\prime \prime}$. 
The diagram which corresponds to the cut vertex $P_{j}$ is one of the atom diagrams of $B_{a}$ which are in $\Delta^{\prime}$. The rest of the proof now follows as in the case where $\breve{\Pi}(D)$ is 2-connected.

Example. Consider the following 3-component link $L$ with its diagram $D$ :
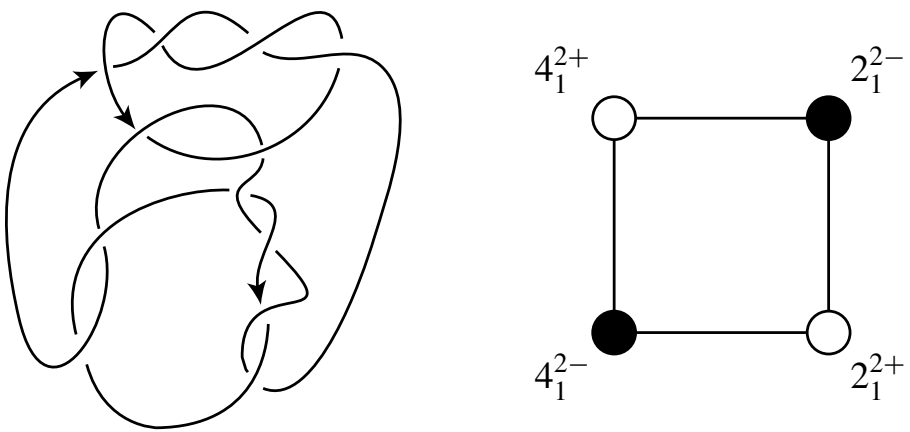

Its graph $\Pi(D)$ is 2-connected. The link $L$ can be considered as a Murasugi sum of a 6 -crossing link which is the connected sum of a $2_{1}^{2+}$ and a $4_{1}^{2+}$ with its mirror image. It can be also considered as a Murasugi sum of the prime knot $6_{2}^{+}$with its mirror image.

Remark 5.6. The simplicity of the proof of Theorem 5.2 when $\Pi(D)$ is a tree made one of us (Quach) believe very early that the theorem could be true in general. But if circuits are present in $\Pi(D)$ it is possible that $\breve{\Phi}$ has no fixed points. It is here that graph theory comes to the rescue. Circuits are organized in bricks and the graph of bricks is a tree. As the theorem is true when $\Pi(D)$ is 2-connected, the general case follows. A fixed point is absolutely needed in our proof, because it indicates where the plumbing Seifert circle is to be found.

\section{Acknowledgements}

We wish to thank Morwen Thistlethwaite for several interesting conversations, and the Fonds National Suisse de la Recherche Scientifique for their support.

\section{References}

[Diestel 2000] R. Diestel, Graphentheorie, Springer-Lehrbuch, Springer, Berlin, 2000. MR 1411445 Zbl 0957.05001

[Gabai 1983] D. Gabai, "The Murasugi sum is a natural geometric operation", pp. 131-143 in Low-dimensional topology (San Francisco, Calif., 1981), Contemp. Math. 20, Amer. Math. Soc., Providence, RI, 1983. MR 85d:57003 Zbl 0524.57004

[Menasco 1984] W. Menasco, "Closed incompressible surfaces in alternating knot and link complements”, Topology 23:1 (1984), 37-44. MR 86b:57004 Zbl 0525.57003 
[Menasco and Thistlethwaite 1993] W. Menasco and M. Thistlethwaite, "The classification of alternating links", Ann. of Math. (2) 138:1 (1993), 113-171. MR 95g:57015 Zbl 0809.57002

[Murasugi 1965] K. Murasugi, “On a certain numerical invariant of link types”, Trans. Amer. Math. Soc. 117 (1965), 387-422. MR 30 \#1506 Zbl 0137.17903

[Quach Hongler and Weber 2004] C. V. Quach Hongler and C. Weber, "On the topological invariance of Murasugi special components of an alternating link", Math. Proc. Cambridge Philos. Soc. 137:1 (2004), 95-108. MR 2005h:57011 Zbl 1057.57007

[Thompson 1994] A. Thompson, “A note on Murasugi sums”, Pacific J. Math. 163:2 (1994), 393395. MR 94k:57018 Zbl 0809.57003

Received January 10, 2004. Revised January 13, 2005.

\section{CAM VAN QUaCh Hongler}

SECTION DE MATHÉMATIQUES

UNIVERSITÉ DE GENÈVE

CP 64

CH-1211 GENÈVE 4

SWITZERLAND

cam.quach@math.unige.ch

Claude Weber

SECTION DE MATHÉMATIQUES

UNIVERSITÉ DE GENÈVE

CP 64

CH-1211 GENÈVE 4

SWITZERLAND

claude.weber@math.unige.ch 